\title{
Oral Taodan granules for mild-to-moderate psoriasis vulgaris: protocol for a randomized, double-blind, multicenter clinical trial
}

\author{
Yi Ru ${ }^{1 \#}$, Xiao-Ning Yan ${ }^{2 \#}$, Su-Qing Yang ${ }^{3}$, Li-Ping Gong ${ }^{4}$, Ling-E Li ${ }^{5}$, Jie Chen ${ }^{1}$, Yi-Ding Zhao ${ }^{2}$, \\ Yue-Peng An ${ }^{3}$, Gang Huang ${ }^{4}$, Jin-Fang Zhang ${ }^{5}$, Qing-Feng Yin ${ }^{6}$, Rui-Ping Wang ${ }^{7}, \mathrm{Xin} \mathrm{Li}^{1,8}$, Bin $\mathrm{Li}^{1,2,8}$ \\ ${ }^{1}$ Department of Dermatology, Yueyang Hospital of Integrated Traditional Chinese and Western Medicine, Shanghai University of Traditional \\ Chinese Medicine, Shanghai 200437, China; ${ }^{2}$ Department of Dermatology, Shaanxi Hospital of Traditional Chinese Medicine, Xi'an 710003, \\ China; ${ }^{3}$ Department of Dermatology, First Affiliated Hospital of Heilongjiang University of Traditional Chinese Medicine, Harbin 150040, China; \\ ${ }^{4}$ Department of Dermatology, the Affiliated Hospital of Jiangxi University of Traditional Chinese Medicine, Nanchang 330006, China; ${ }^{5}$ Department \\ of Dermatology, Traditional Chinese Medicine Hospital of Shijiazhuang, Shijiazhuang 050051, China; ${ }^{6}$ Jiangsu Famous Medical Technology Co. \\ Ltd., Nanjing University of Traditional Chinese Medicine, Nanjing 210029, China; ${ }^{7}$ Office of National Clinical Research Base of TCM, Yueyang \\ Hospital of Integrated Traditional Chinese and Western Medicine, Shanghai University of Traditional Chinese Medicine, Shanghai 200437, China; \\ ${ }^{8}$ Institute of Dermatology, Shanghai Academy of Traditional Chinese Medicine, Shanghai 201203, China \\ Contributions: (I) Conception and design: X Li, B Li; (II) Administrative support: B Li, XN Yan; (III) Provision of study materials or patients: YD \\ Zhao, YP An, G Huang, JF Zhang, J Chen; (IV) Collection and assembly of data: SQ Yang, LP Gong, LE Li; (V) Data analysis and interpretation: \\ QF Yin, RP Wang; (VI) Manuscript writing: All authors; (VII) Final approval of manuscript: All authors. \\ \#These authors contributed equally to this work. \\ Correspondence to: Xin Li; Bin Li. Department of Dermatology, Yueyang Hospital of Integrated Traditional Chinese and Western Medicine, Shanghai \\ University of Traditional Chinese Medicine, No. 110 Ganhe Road, Hongkou District, Shanghai 200437, China. Email: 13661956326@163.com; \\ 18930568129@163.com.
}

Background: Psoriasis is a common chronic inflammatory skin disease with high recurrence rates and increasing incidence. Patients require long-term medication to reduce symptoms and prevent disease progression. Therefore, the development of treatments with high efficiency and low rate of adverse events is of utmost importance. Traditional Chinese medicine (TCM) plays an outstanding role in reducing disease symptoms and improving quality of life. The aim of this trial is to clarify the treatment efficacy, safety, and control of disease recurrence in patients with psoriasis with blood-stasis syndrome treated with Taodan granules (TDKL).

Methods: This trial is a five-center, randomized, double-blind, placebo-controlled study planned to transpire between September 1, 2019, and December 31, 2021. A sample size of 216 participants (108 per group) with mild-to-moderate psoriasis will be randomly assigned to receive TDKL or placebo twice per day, 7 days per week, for 8 weeks. The study duration will be 17 weeks, including a 1 -week screening period, 8 weeks of intervention, and another 8 weeks of follow-up. The primary outcomes are improvement in the Psoriasis Area and Severity Index score and recurrence rate after 8 weeks of treatment. Secondary outcomes include body surface area affected and the scores for the Physician Global Assessment, Dermatology Life Quality Index, pain-related quality of life, pain on the visual analogue scale, and TCM syndromes. The number, nature, and severity of adverse events will be carefully recorded.

Discussion: The study results will help clarify the safety and efficacy of TDKL as treatment for psoriasis with respect to both disease regression and recurrence rate. We expect that this study will provide highquality evidence with important public health implications that may alter the approach to psoriasis management in China.

Trial registration: The trial has been registered at ClinicalTrials.gov (ID: NCT03942198).

Keywords: Promoting blood circulation and removing blood stasis (PBCRBS); psoriasis; randomized controlled trial (RCT); Taodan granule (TDKL); traditional Chinese medicine (TCM) 
Submitted Aug 01, 2019. Accepted for publication Aug 20, 2019.

doi: 10.21037/atm.2019.09.05

View this article at: http://dx.doi.org/10.21037/atm.2019.09.05

\section{Introduction}

Psoriasis is a chronic, relapsing, inflammatory, multisystem disease characterized by infiltration of inflammatory cells (especially $\mathrm{T}$ cells), hyperplasia of epidermal keratinocytes, and abnormal differentiation (1). While the pathogenesis of psoriasis remains unknown, recent studies (2) recognize many contributing factors such as genetics, environment, immune tolerance, inflammation, and metabolism. In the past few years, the global prevalence of psoriasis ranged from $0.51 \%$ to $11.43 \%$ in adults and from $0 \%$ to $1.37 \%$ in children (3). However, the incidence of psoriasis has been increasing considerably (4), suggesting inadequate prevention and treatment of this condition.

Plaque psoriasis represents the most common manifestation, accounting for approximately $80 \%$ of psoriasis cases, and more than $90 \%$ of patients are diagnosed in the chronic phase, requiring long-term treatment (5). The treatment of psoriasis involves the use of topical agents, ultraviolet light, systemic drugs, and/or biologics. Because systemic drugs have serious side effects related to organ toxicity and biologics increase the risk of infections, such medicines are mainly used to treat severe psoriasis $(6,7)$. Topical therapy is the most common treatment for mildto-moderate psoriasis, providing maximum control over the area affected by the drug, with minimal systemic effects (8). In addition to these conventional approaches, traditional Chinese medicine (TCM) has also attracted widespread attention, owing to its efficacy and lower incidence of side effects.

TCM has shown long-lasting therapeutic effect in psoriasis, providing adequate disease control with few adverse events (AEs) and minimal toxicity (9). Within the TCM theory, psoriasis is considered to be caused by "blood heat" and the stagnation of qi and blood. Previous studies have shown significant changes in immune serum parameters in patients with psoriasis with blood-heat syndrome (10). Long-term blood heat can lead to blood stasis, poor circulation of qi and blood, and blockage of meridians and collaterals, depriving the skin of nutrients and hydration, thus leading to psoriasis. The main TCM treatment for psoriasis caused by the blood-heat syndrome consists of removing heat and cooling the blood, as well as promoting blood circulation and removing blood stasis (PBCRBS). Liu et al. (11) reported that activating the blood circulation could improve immune function and blood clearing, which helps treat psoriasis. The Yinxieling (12) formula has been used in the treatment of psoriasis with blood-stasis syndrome and has been proven effective in decreasing the Psoriasis Area and Severity Index (PASI) scores in the clinical setting.

Taodan granules (TDKL), a Chinese herbal prescription, is a compound preparation with nine ingredients (milkvetch, licorice, angelica, rhizoma chuanxiong, peach seed, Salvia miltiorrbiza, Curcuma zedoaria, Achyranthes bidentata, and sarsaparilla) (Table 1). The predecessor of TDKL is Xia's Huoxue Decoction, which was discovered in the 1950s by Xia Han (a well-known Chinese surgeon) and has been used in the clinical treatment of psoriasis for over 50 years at the Yueyang Hospital of Integrated Traditional Chinese and Western Medicine. According to the syndrome differentiation approach in TCM, there are three syndromes of psoriasis: blood stasis, blood heat, and blood dryness. Therefore, the TCM approach for the treatment of psoriasis is based on ameliorating these blood syndromes, starting with blood heat and continuing with blood stasis. The theory of "hemo-modulating therapy" is widely applied and guides the current clinical practice of TCM-based treatment of psoriasis. TDKL has PBCRBS properties and thus it is often used in the treatment of psoriasis with bloodstasis syndrome $(13,14)$.

Recent studies revealed a relationship between Th cell differentiation and different syndromes of psoriasis, as defined within TCM, suggesting that different symptoms reflect different immune states (15). Preliminary research (16) revealed that the ratio of Treg/Th17 cells in peripheral blood and the expression levels of major cytokines and transcription factors differed across patients with different syndromes of psoriasis. Recent data from clinical studies confirm the effectiveness of TDKL in psoriasis (13), with $76.64 \%$ of lesions showing improvement in the affected area and in the PASI score. Furthermore, TDKL has a significant effect on the expression levels of epidermal cytokines (14). However, due to the complex composition of TDKL, the mechanism underlying its therapeutic action remains unclear. 
Table 1 Ingredients of Taodan granules (intervention drug) with English translations

\begin{tabular}{llll}
\hline Main composition & Chinese Pinyin & Latin scientific name & Plant part \\
\hline Milkvetch & Huang Qi & Astragalus adsurgens Pall. & Root \\
Licorice & Gan Cao & Glycyrrhiza glabra & Root \\
Angelica & Dang Gui & Angelica sinensis radix & Root \\
Rhizoma chuanxiong & Chuan Xiong & Ligusticum wallichii & Rhizome \\
Peach seed & Tao Ren & Prunus persica (L.) Batsch & Seed \\
Chinese sage & Dan Shen & Salvia miltiorrhiza Bunge & Root \\
Zedoary & E Zhu & Curcuma zedoaria (Christm.) Rosc. & Rhizome \\
Ox knee & Chuan Niu Xi & Achyranthes bidentata Blume & Root \\
Sarsaparilla & Ba Qia & Smilax china (L.) & Rhizome \\
\hline
\end{tabular}

The beneficial effect of TDKL in psoriasis is widely known and considered to originate from amelioration of the blood-stasis syndrome, leading to symptom improvement and lack of disease progression. However, no large-scale randomized controlled trials have been conducted on the TDKL treatment efficacy, safety, and control of disease recurrence. Therefore, we aim to conduct a multicenter, randomized, double-blind, placebo-controlled clinical trial of oral TDKL (PBCRBS) treatment for psoriasis with blood-stasis syndrome.

\section{Methods}

\section{Design}

We designed this study as a multi-center, randomized, double-blind, placebo-controlled clinical trial. The aim is to clarify the clinical efficacy, safety, and control of disease recurrence in patients with psoriasis with bloodstasis syndrome. The results of the study will be useful to establish a clinical standard for the treatment of psoriasis. The trial has been registered at ClinicalTrials.gov (ID: NCT03942198). At the time of writing of the trial protocol (version 3.0, May 30, 2019), the trial had yet to start enrollment. The start and end of recruitment had been planned to be September 1, 2019, and December 31, 2021, respectively (Figure S1). This protocol has been developed according to Standard Protocol Items: Recommendations for Interventional Trials (SPIRIT) 2013 (17,18). The checklist is described in detail in Table S1.

Prior to trial initiation, the investigators and researchers are conducting unified training to ensure that the medical staff involved in the study fully understand all aspects of the trial. The study will be conducted in five clinical centers across China, namely the Shaanxi Hospital of TCM, Yueyang Hospital of Integrated Traditional Chinese and Western Medicine, the Affiliated Hospital of Jiangxi University of TCM, TCM Hospital of Shijiazhuang, and First Affiliated Hospital of Heilongjiang University of TCM. Competitive enrollment will be applied in all research centers, to achieve the goal of 216 participants in total. Each participant can only be enrolled once.

The study will consist of five phases: screening/ enrollment, allocation, treatment/intervention, end of intervention, and follow-up. During enrollment, participants will be recruited from the dermatology clinic setting, where they will undergo physical examination and assessment of eligibility. The maximum time allowed between the assessment and the intervention is 1 week (week 0). If more than a week has passed since the assessment, the participant will have to undergo repeat assessment before being allowed to start the intervention. Eligible participants will be invited to sign the written informed consent regarding participation in the trial (procedures, risks, options for dropping out), regarding the use of laboratory data, and regarding the collection, storage, and use of biological specimens. A study investigator or medical staff member that has received adequate training will provide the participant with extensive explanations. Upon agreeing to provide consent, the participant will be invited to sign and date the informed consent form, at which time a participant identification (PID) number will be assigned to facilitate PID throughout the study.

The participants will be randomly assigned to 


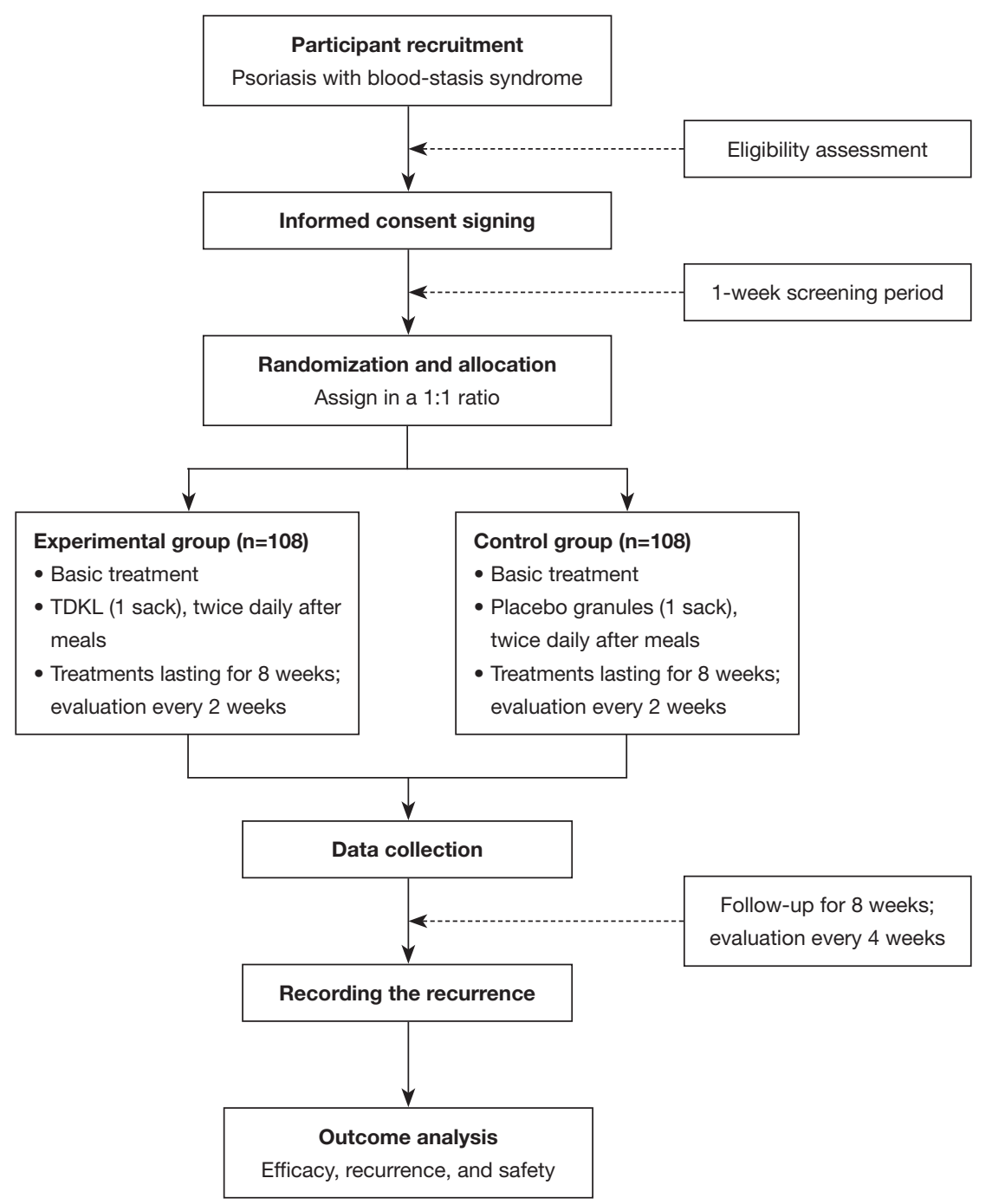

Figure 1 Flow diagram of the progress through the study. TDKL, Taodan granules.

the experimental or control group and undergo the corresponding intervention for a total of four treatment cycles. Participants in the experimental group will receive TDKL, whereas those in the control group will receive placebo granules (Figure 1). The participants will continue to receive systemic therapy, as judged by their treating physician. All changes in symptoms, prescriptions, relevant scores, and macroscopic characteristics (based on photographic evidence), along with any AEs, will be recorded.

\section{Randomization and allocation}

This study uses a center-based stratification and block randomization method. The randomization sequence will be generated by study investigators who are statisticians. Patients will be allocated in a 1:1 ratio, aiming to balance baseline characteristics between the groups. Participants will be assigned a PID number, which will be used for subject identification throughout the study. Information regarding the random-number block will be delivered to the participating centers along with the intervention drugs.

\section{Sample size}

The required sample size was calculated using an estimation formula based on the difference between two sample rates (19). A previous clinical study (13) reported that 
$55.66 \%$ of patients taking Chinese herbal medicine and $35.3 \%$ of patients taking placebo achieved $50 \%$ PASI reduction. Setting the two-sided significance level $(\alpha)$ at 0.05 and statistical power at 0.8 , a minimum sample size of 90 participants per group (180 participants in total) was estimated to provide sufficient statistical power for detecting a between-group difference of $20 \%$ in treatment efficiency, defined as the change in PASI score. Considering a $20 \%$ loss to follow-up, we aim to enroll 108 patients in each arm, for a total of 216 patients.

\section{Eligibility criteria}

The following inclusion criteria will be applied at the time of randomization: (I) diagnosis of psoriasis established in accordance with the conventional diagnostic criteria for psoriasis, as well as in accordance with the syndrome diagnostic criteria within TCM; (II) mild-to-moderate disease severity, defined as skin lesions covering $\leq 10 \%$ of the body surface area (BSA); (III) age between 18 and 65 years; (IV) written informed consent. Eligible participants will have to fulfill all inclusion criteria.

The following exclusion criteria will be applied: (I) erythrodermic, arthritic, pustular, or punctate psoriasis; (II) other active skin disease that may affect assessment; (III) prescription for research drugs, biological agents, or immunosuppressive agents within the month leading up to the study; (IV) prescription for topical glucocorticoids, phototherapy, or other relevant therapies within the 2 weeks leading up to the study; (V) severe or uncontrollable local/systemic acute/chronic infection; (VI) severe systemic disease or abnormal laboratory values; (VII) history of malignant tumors or primary/secondary immunodeficiency or hypersensitivity; (VIII) major surgery during the 8-week study period; (IX) pregnancy or breastfeeding; (X) history of alcoholism or drug abuse; (XI) personal or family history of serious mental illness; (XII) any other serious medical condition judged by the researchers to pose a significant risk to the patient or interfere with the interpretation of study outcomes. Candidates satisfying one or more exclusion criteria will be excluded.

\section{Interventions}

\section{TDKL intervention}

Participants in the experimental group will receive 112 sacks of experimental granules over the course of 8 -week treatment period and will be instructed to consumed one sack in the morning and one in the evening at approximately 30 minutes after meals (two sacks per day, 7 days per week, for 8 weeks). The major ingredients of TDKL are listed in Table 1 .

\section{Intervention}

Participants in the control group will receive 112 sacks of placebo granules over the course of 8-week treatment period and will be instructed to consumed one sack in the morning and one in the evening at approximately 30 minutes after meals (the same protocol as that used in the experimental group).

\section{Basic treatment}

All participants will receive basic treatment with Dr. Yu moisturizing cream (Shanghai Jahwa United Co., Ltd., Shanghai, China) to be applied within 2 minutes after bathing in order to avoid dehydration. The participants will be instructed to continue their daily activities and maintain communication with their treating physician, as well as with the staff involved in the study.

\section{Treatment cycles}

The 8 -week treatment period was planned in four cycles of 2 weeks, with a return visit between cycles to check for AEs and monitor compliance. All interventions will be stopped after 8 weeks. Follow-up will be conducted on weeks 12 and 16.

\section{Intervention drugs and blinding}

All intervention drugs (TDKL in the experimental group and placebo granules in the control group) will be supplied by China Resources Sanjiu Medical \& Pharmaceutical Co., Ltd. (Shenzhen, China) under a unified packaging and labeling format. The placebo granules will simulate the same appearance and taste of TDKL.

The study is designed as a double-blind investigation. The participants, study monitors, and study investigators will be blinded throughout the duration of the study. The PID will be the only information linked to group allocation. Concealment will be ensured by the fact that the randomization code will be maintained by the Data Management Center of Jiangsu Famous Medical Technology Co. Ltd.

For the purpose of statistical analysis, the TDKL intervention will be considered the exposure. In the event of 
serious AEs, the principal investigators handling the study at each participating center will decide whether breaking allocation concealment is required. If concealment is broken before the end of the study, the data will be censored for the purpose of analysis and the patient considered to have dropped out of the study.

\section{Drug combination}

All drugs used in the study will be considered combined drugs and their use will have to be recorded in the case report form. The trade name, dosage, indications, and duration of medication will be recorded. The study investigators or researchers will judge whether the participant should withdraw from the study on account of the nature of the combined drugs. However, the use of steroid hormones during the study period shall not be allowed.

\section{Outcome evaluation}

\section{Primary outcome}

The primary outcomes of this trial are improvement in the PASI score and disease recurrence rate after 8 weeks of treatment. The PASI score will be assessed every 2 weeks during the treatment period and every 4 weeks during the follow-up period. All participants who complete the 8-week treatment period will be followed up for another 8 weeks (from week 9 to week 16). Relapse is defined as a PASI score decrease of more than $50 \%$ by week 12 or week 16 versus the maximum value noted since enrollment.

\section{Secondary outcome}

Secondary outcomes include affected BSA and the scores for Physician Global Assessment (PGA), Dermatology Life Quality Index (DLQI), pain-related quality of life (PRQoL), pain on the visual analogue scale (VAS), and TCM syndromes, all of which will be assessed every 2 weeks during the treatment period. During the follow-up period, BSA, PGA scores, and VAS scores will be evaluated every 4 weeks, whereas the DLQI, PRQoL, and TCM syndrome scores will only be evaluated on week 16 . Laboratory data will be monitored until the last visit (Table 2).

\section{Safety evaluation}

The Chinese herbal medicines contained in TDKL are all listed in the Pharmacopoeia of the People's Republic of China. The dose used in this study is within the range recommended by the Pharmacopoeia Commission of the Ministry of Health of the People's Republic of China. To date, no adverse reactions have been reported in relation to the clinical application of TDKL. Nevertheless, the trial will implement adequate measures to monitor for AEs, including observation of vital signs, laboratory tests, recording of concomitant medications, pregnancy tests, and physical examinations. All AEs will be recorded, regardless of severity, in order to assess the safety of TDKL. The specific implementation of such measures is reflected in Table 2.

If an $\mathrm{AE}$ occurs, investigators have to determine whether to stop the observation and proceed with the diagnosis and corresponding treatment. If a severe $\mathrm{AE}$ occurs, the study investigator must take immediate action to ensure the safety of the participants. In addition, all severe AEs will be reported to the Shaanxi Hospital of TCM and to the Ethics Committee within 24 hours. The responsible staff from the Shanxi Hospital of TCM shall promptly notify other participating centers and initiate any necessary legal procedures.

\section{Data management and monitoring}

Data associated with this study will be collected and maintained into a dedicated database of research medical records that log outpatient and inpatient data about the participants and must be submitted by the investigators at the end of follow-up (week 16) together with the participant medication records and informed consent forms. The principal investigators at each participating center are responsible for collecting the research medical records from the study investigators, as well as for reviewing and storing these records.

Patient data are entered into electronic case report forms by the responsible staff at each participating center. In order to ensure the quality and consistency of the source data and of the data entered into the database, two researchers will independently check the source data and compare against the information entered into the corresponding electronic case report forms. Any questions or suspicions arising during the process of checking the source and case report data shall be added to a formal list of queries, which will be addressed by the investigator filling out the data. If a problem is found, it should be processed and recorded in a timely manner. All documentation on quality control will be maintained in order to objectively assess safety and key 


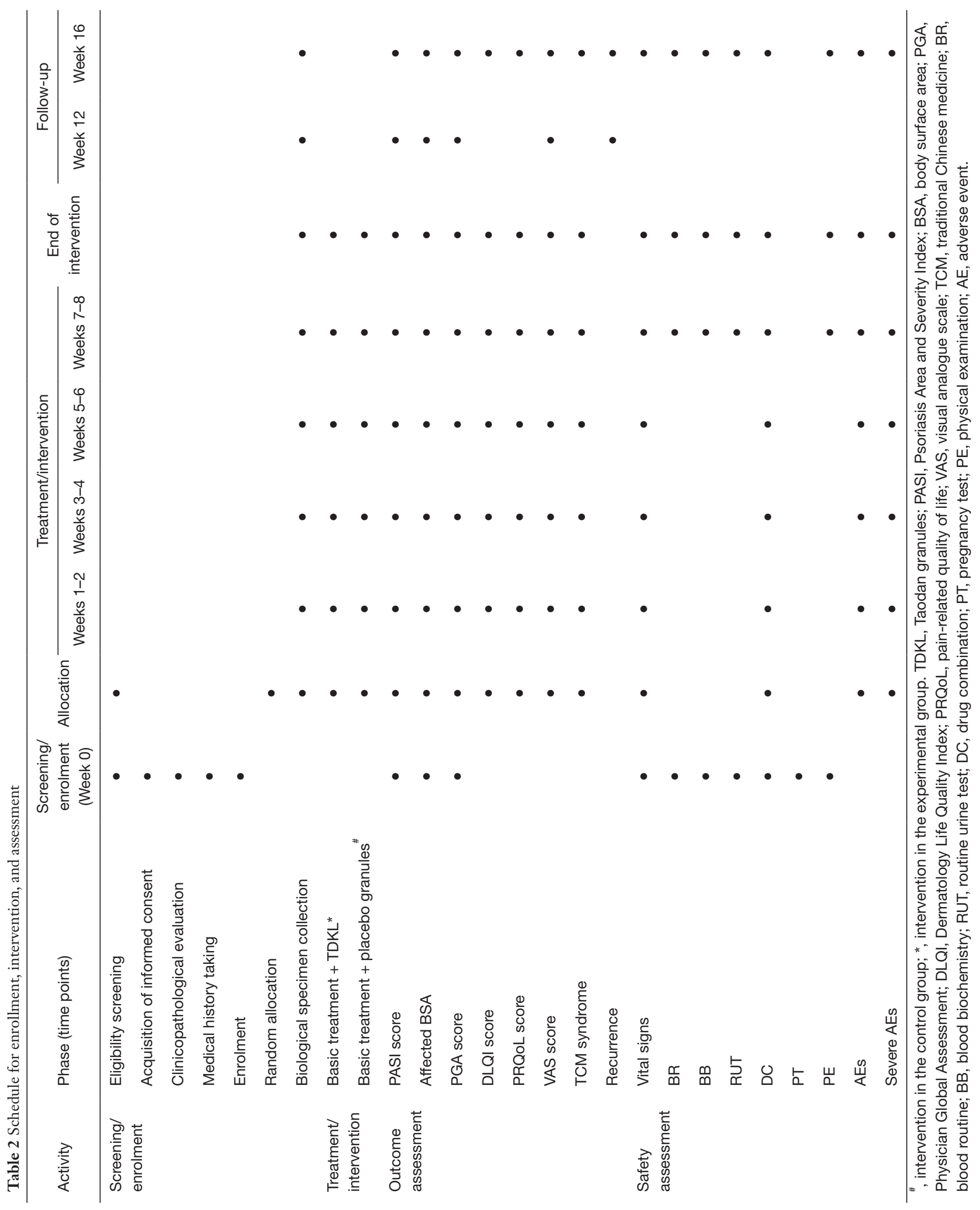


outcomes.

\section{Statistical analysis}

The statistical analysis plan was developed by professional statisticians upon consultation with the main trial investigators. The data will be stored with the Data Management Center of Jiangsu Famous Medical Technology Co. Ltd., and processed by their in-house statisticians blinded to group allocation. The analyses will be conducted using SAS version 9.2 (SAS Institute Inc., Cary, NC, USA) and will cover the number of participants enrolled in each group, the number of patients who dropped out of the study and the reason for the drop-out, demographic and other baseline characteristics, compliance, efficacy analysis, and safety analysis.

Descriptive and comparative analyses will be conducted. Qualitative data will be described using frequency tables, percentages, or constituent ratios and compared using the chi-square test, Fisher's exact probability test, Wilcoxon rank-sum test, Cochran-Mantel-Haenszel chi-square test and weighted least squares covariance. Quantitative data will be described using the mean, standard deviation, median, quartile thresholds, minimum, and maximum. The $t$-test will be used for comparative analysis of data with normal distribution, with Satterthwaite correction if the variance is uneven. Quantitative data exhibiting non-normal distribution on the Wilcoxon rank-sum test will be analyzed using the Wilcoxon signed-rank sum test and covariance generalized linear models. All hypothesis tests will be twosided. The test statistics and corresponding $\mathrm{P}$ values will be reported. Statistical significance will be established at $\mathrm{P}<0.05$, with high statistical significance established at $\mathrm{P}<0.01$.

\section{Discussion}

Psoriasis is a multi-system disease involving abnormal immune response. The course of the disease is long and the symptoms recur frequently, requiring long-term medication. At present, topical therapy remains the firstline treatment for mild-to-moderate psoriasis vulgaris. Additionally, TCM is widely applied due to its suitability for long-term use at reasonable doses $(20,21)$. There is increasing evidence that psoriasis not only affects the skin but also reflects chronic multi-system inflammation. Indeed, patients with psoriasis often have other comorbidities including obesity, myocardial infarction, atherosclerosis, diabetes mellitus, renal disease, or mild liver disease (22-24). The risk of comorbidities varies according to the type of psoriasis, supporting the application of the TCM "hemo-modulating therapy" in the treatment of psoriasis with blood-stasis syndrome.

TCM considers that chronic illness leads to blood stasis, which accumulates in the body and causes disease, thus promoting the persistence and aggravation of chronic disease. Hence, the PBCRBS approach has always been the focus of TCM-based treatment and prevention of psoriasis. PBCRBS should be promoted throughout the duration of treatment for psoriasis. In addition, blood stasis in the meridians, viscera, and limbs leads to a variety of comorbid syndromes that resemble metabolic diseases. Therefore, in this trial of psoriasis with blood-stasis syndrome, we selected a Chinese medicine with PBCRBS action.

PBCRBS agents such as TDKL can relieve pain by activating blood circulation, which removes blood stasis and disperses stagnant blood (25). Tanshinone IIA, an active component of TDKL originating from Salvia miltiorrbiza material, can inhibit keratinocyte proliferation, induce keratinocyte apoptosis, and block the cell cycle in a concentration- and time-dependent manner (26). Based on these previous observations, we cultured human immortal keratinocyte cells with serum containing TDKL and found that that TDKL could inhibit cell proliferation (data not shown), which supports the therapeutic effect of TDKL on psoriasis with blood-stasis syndrome and has motivated us to initiate this trial.

In this study, we plan to use the granule formulation in order to minimize bias associated with the use of herbs from different geographical areas, of different varieties, and maintained under different storage conditions. In addition, due to the multi-center study design in the clinical setting, the outcomes of this research are expected to provide more general clinical evidence that implicitly accounts for the impact of environmental factors and individual patient characteristics on the outcome of the trial. In practice, patients often continue to apply medicine they found effective in order to consolidate the efficacy even after full symptom regression. However, there is yet no evidence to support this practice. In the present study, we aim to clarify the short-term efficacy of TDKL treatment twice per day, every day, for 8 weeks. As oral preparations of PBCRBS herbs sometimes exhibit side effects such as aggravating preexisting conditions (bleeding tendency, menstrual disorders, damage to the gastric mucosa), special emphasis has been placed upon planning for at least 4 weeks of follow-up, to 
cover at least one menstrual cycle.

We will carefully monitor for recurrence of psoriasis after treatment completion. Additionally, we will record all AEs and information regarding concomitant medications at each visit. The study results will help clarify the safety and efficacy of TDKL as treatment for psoriasis with respect to both disease regression and recurrence rate. Therefore, this trial represents an important opportunity to test the validity of the theory underlying the TCM "hemo-modulating therapy" in psoriasis treatment. We expect that this study will provide high-quality evidence that can be used for developing clinical treatment guidelines. Therefore, the results of this study are expected to have important public health implications that may even alter the approach to psoriasis management in China.

The present study has limitations that should be noted. Only one regimen for the experimental drug will be tested and the prescription will not be adapted based on symptom differentiation; hence, the findings might be specific to the tested regimen. Nevertheless, the findings will still be useful as reference in clinical practice and will help pave the way for future research. Finally, the TDKL prescription to be used in this trial was designed for the treatment of psoriasis with blood-stasis syndrome; thus, the findings may not be applicable to other psoriasis syndromes.

\section{Acknowledgments}

Funding: This study was supported by a grant from the National Key Research and Development Program of People's Republic of China (grant number 2018YFC1705303) and by the National Natural Science Foundation of China (grant numbers 81973860, 81874470), Hundred Talents Program of Shaanxi Province of China (grant number SXBR9053), and Shanghai Development Office of TCM [grant numbers ZY(2018-2020)FWTX-1008, ZY(2018-2020)-CCCX-2004-08, ZY(20182020)-FWTX-4010].

\section{Footnote}

Conflicts of Interest: The authors have no conflicts of interest to declare.

Ethical Statement: The authors are accountable for all aspects of the work in ensuring that questions related to the accuracy or integrity of any part of the work are appropriately investigated and resolved. Ethical approval has been obtained from the Ethics Committee of the Yueyang Hospital of Integrated Traditional Chinese and Western Medicine (approval no. 2019-032) (Figure S2). All participants will be enrolled only after providing written informed consent. No clinical data or bio-samples will be collected without the participants' consent. The trial is conducted in accordance with national laws, Good Clinical Practice (GCP) guidelines, and the Declaration of Helsinki as revised in 2013 (Table S1). The results will be disseminated to the public through conference presentations and papers in open access journals. All authors are accountable for all aspects of the work and will ensure the integrity and accuracy of any part of the work.

\section{References}

1. Albanesi C, Madonna S, Gisondi P, et al. The Interplay Between Keratinocytes and Immune Cells in the Pathogenesis of Psoriasis. Front Immunol 2018;9:1549.

2. Lewis DJ, Chan WH, Hinojosa T, et al. Mechanisms of microbial pathogenesis and the role of the skin microbiome in psoriasis: A review. Clin Dermatol 2019;37:160-6.

3. Michalek IM, Loring B, John SM. A systematic review of worldwide epidemiology of psoriasis. J Eur Acad Dermatol Venereol 2017;31:205-12.

4. Parisi R, Symmons DP, Griffiths CE, et al. Global epidemiology of psoriasis: a systematic review of incidence and prevalence. J Invest Dermatol 2013;133:377-85.

5. Nevitt GJ, Hutchinson PE. Psoriasis in the community: prevalence, severity and patients' beliefs and attitudes towards the disease. Br J Dermatol 1996;135:533-7.

6. Raaby L, Zachariae C, Ostensen M, et al. Methotrexate Use and Monitoring in Patients with Psoriasis: A Consensus Report Based on a Danish Expert Meeting. Acta Derm Venereol 2017;97:426-32.

7. Doherty SD, Van VA, Lebwohl MG, et al. National Psoriasis Foundation consensus statement on screening for latent tuberculosis infection in patients with psoriasis treated with systemic and biologic agents. J Am Acad Dermatol 2008;59:209-17.

8. Prussick R, Wu JJ, Armstrong AW, et al. Psoriasis in solid organ transplant patients: best practice recommendations from The Medical Board of the National Psoriasis Foundation. J Dermatolog Treat 2018;29:329-33.

9. Lu CJ, Yu JJ, Deng JW. Disease-syndrome combination clinical study of psoriasis: Present status, advantages, and prospects. Chin J Integr Med 2012;18:166-71.

10. Li X, Xiao QQ, Li FL, et al. Immune Signatures in 
Patients with Psoriasis Vulgaris of Blood-Heat Syndrome: A Systematic Review and Meta-Analysis. Evid Based Complement Alternat Med 2016;2016:9503652.

11. Liu H, Tan Q, Liu H. A clinical study on treatment of senile psoriasis by replenishing qi to activate blood--a report of 40 cases. J Tradit Chin Med 2004;24:204-7.

12. Yan YH, Lu CJ. Optimized Yinxieling for treatment of psoriasis vulgaris: an exploratory clinical trial. Tradit Chin Drug Res Clin Pharmacol 2011;22:691-3.

13. Fan B, Li B, Zhang Y, et al. Changes of SP and Betaendorphin in Active and Quiescent Period of Psoriasis Patients Intervened by Blood-Cooling and BloodActivating Herbs. Shanghai Journal of Traditional Chinese Medicine 2006;40:34-5.

14. Fan B, Li B, Shen JX, et al. Effects of TCM Bloodcooling and Hemo-activating Formula on Cytokine Production of Psoriasis Patients in different Periods. Chin J Dermatovenereol Integr Tradit West Med 2006;5:70-1.

15. Chen J, Cao XX, Xu R, et al. Research on different expressions of peripheral blood Th1/Th2 cells in psoriasis patients of blood heat syndrome and of blood stasis syndrome. Zhongguo Zhong Xi Yi Jie He Za Zhi 2014;34:46-50.

16. Fan B, Li X, Ze K, et al. Expression of T-helper 17 cells and signal transducers in patients with psoriasis vulgaris of blood-heat syndrome and blood-stasis syndrome. Chin J Integr Med 2015;21:10-6.

17. Wang JZ, Li YY, Chen GM. Medical statistics. Zhengzhou: Zhengzhou University Press, 2002:112.

18. Chan AW, Tetzlaff JM, Altman DG, et al. SPIRIT 2013 statement: defining standard protocol items for clinical

Cite this article as: Ru Y, Yan XN, Yang SQ, Gong LP, Li LE, Chen J, Zhao YD, An YP, Huang G, Zhang JF, Yin QF, Wang RP, Li X, Li B. Oral Taodan granules for mild-to-moderate psoriasis vulgaris: Protocol for a randomized, double-blind, multicenter clinical trial. Ann Transl Med 2019;7(18):488. doi: 10.21037/atm.2019.09.05 trials. Ann Intern Med 2013;158:200-7.

19. Moher D, Schulz KF, Altman DG, et al. The CONSORT statement: revised recommendations for improving the quality of reports of parallel-group randomized trials. JAMA 2001;285:1987-91.

20. Zheng Q, Jiang WC, Sun XY, et al. Total glucosides of paeony for the treatment of psoriasis: A systematic review and meta-analysis of randomized controlled trials. Phytomedicine 2019;62:152940.

21. Chen Y, Guo DJ, Deng H, et al. Acute and chronic toxicity of a polyherbal preparation-Jueyin granules. BMC Complement Altern Med 2018;18:148.

22. Agca R, Heslinga SC, Rollefstad S, et al. EULAR recommendations for cardiovascular disease risk management in patients with rheumatoid arthritis and other forms of inflammatory joint disorders: 2015/2016 update. Ann Rheum Dis 2017;76:17-28.

23. Li X, Miao X, Wang H, et al. Association of Serum Uric Acid Levels in Psoriasis: A Systematic Review and MetaAnalysis. Medicine (Baltimore) 2016;95:e3676.

24. Yeung H, Takeshita J, Mehta NN, et al. Psoriasis severity and the prevalence of major medical comorbidity: a population-based study. JAMA Dermatol 2013;149:1173-9.

25. Lu X, Li B. Exploration of the effect and mechanism of activating blood circulation and stasis-removing therapy on tumor metastasis. Chin J Integr Med 2009;15:395-400.

26. Li FL, Xu R, Zeng QC, et al. Tanshinone IIA Inhibits Growth of Keratinocytes through Cell Cycle Arrest and Apoptosis: Underlying Treatment Mechanism of Psoriasis. Evid Based Complement Alternat Med 2012;2012:927658. 


\section{Supplementary}

课题编号: 2018YFC1705303

国家重点研发计划 课题任务书
National Key Research and Development Program of China Task book

\section{课题名称：活血散疼法治疗银屑病血瘀证多中心随机对照研究}

所属项目: 银屑病”新血证论”理论体系构建与实践

所属专项: 中医药现代化研究

项目牵头承担单位: 上海中医药大学附属岳阳中西医结合医院

课题承担单位: 陕西省中医医院

课题负责人: 间小宁

执行期限： 2019 年 01 月 至 2021 年 12 月

中华人民共和国科学技术部制

2018 年 12 月 20 日

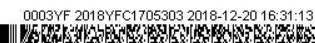

I.
Project Name: Active blood and resolve stasis method for the treatment of psoriasis

Project which belonging to:

Special Project

which belonging to:

Project Lead Unit:

Project

undertaking unit:

Project leader:

Execution period: with blood stasis syndrome:A multi-center randomized controlled trial

Modernization of Traditional Chinese Construction and Practice of the Theoretical System of "New Blood Syndrome" in Psoriasis

Yueyang Hospital of Integrated Traditional Chinese and Western Medicine, Shanghai University of Traditional Chinese Medicine

$\frac{\text { Shaanxi Hospital of Traditional Chinese Medicine }}{\text { Xiaoning Yan }}$
of the People's Republic of China December 20, 2018

OO003YF 2018YFC1705303 2018-12-20 16:31:1

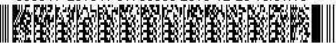

Figure S1 Funding documentation. 


\section{Administrative informatio}

Title

Trial registration

Protocol version

Funding

Roles and responsibilities

Background and rationale

Objectives

Trial design

Methods: participants, interventions, and outcomes

Study setting

Eligibility criteria

Interventions

Outcomes

Participant timeline

Sample size

Recruitment

Methods: assi

Allocation

Sequence generation

Allocation concealment

mechanism

Implementation

Blinding (masking)

Methods: data collection, management, and analysis

Data collection methods

Data management

Statistical methods$$
19
$$

for data values). Reference to where details of data management procedures can be found, if not in the protocol Statistical methods for analysing primary and secondary outcomes. Reference to where other details of the statistical analysis plan can be found if not in the protocol

20b Methods for any additional analyses (e.g., subgroup and adjusted analyses)

$20 \mathrm{c}$ Definition of analysis population relating to protocol non-adherence (e.g., as randomised analysis), and any statistical methods to handle missing data (e.g., multiple imputation)

Methods: monitoring

Data monitoring

21a Composition of data monitoring committee (DMC); summary of its role and reporting structure; statement of whether it is independent from the sponsor and competing interests; and reference to where further details about its charter can be found, if not in the protocol. Alternatively, an sponsor and competing interests; and refe
explanation of why a DMC is not needed

21b Description of any interim analyses and stopping guidelines, including who will have access to these interim results and make the final decision to terminate the trial

Harms

22 Plans for collecting, assessing, reporting, and managing solicited and spontaneously reported adverse events and other unintended effects of trial interventions or trial conduct

Auditing

Ethics and dissemination

Research ethics approval

Protocol amendments

Consent or assent

Confidentiality

Declaration of interests

Access to data

Ancillary and post-trial care

Dissemination policy

Appendices

Informed consent materials $\quad 32$

Biological specimens

23 Frequency and procedures for auditing trial conduct, if any, and whether the process will be independent from investigators and the sponsor 

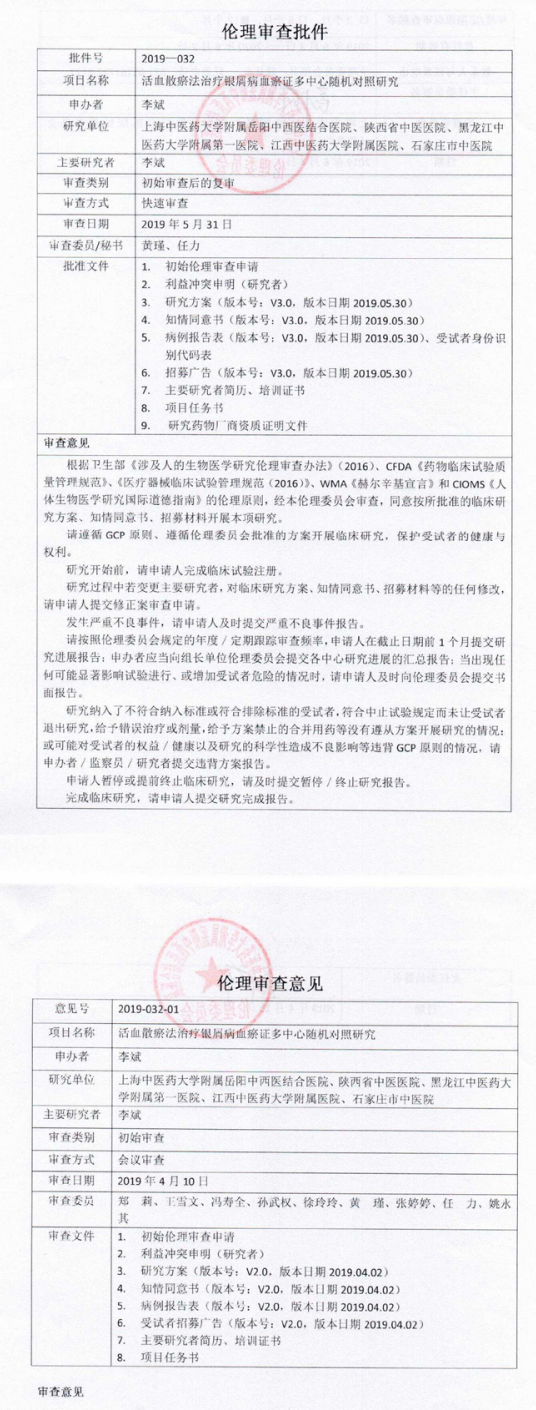

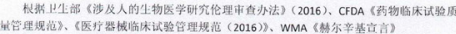

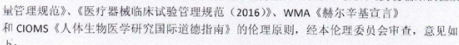

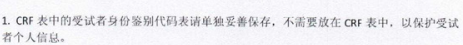

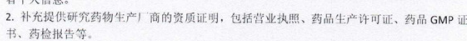

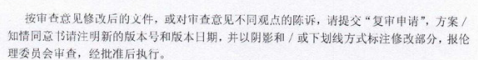

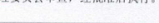

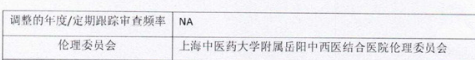

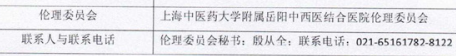

Figure S2 Ethical approval document.
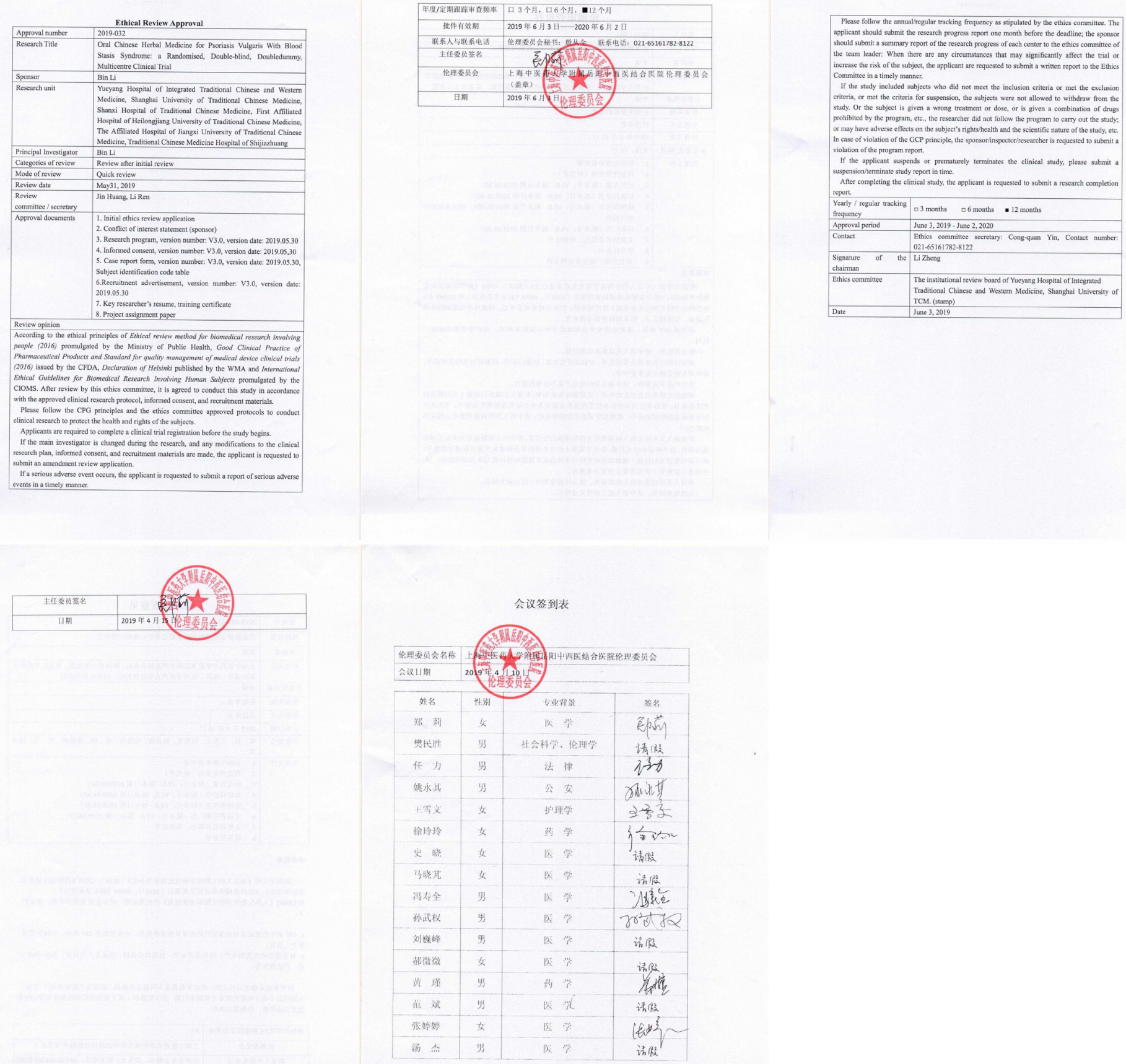